Published in final edited form as:

Vet Radiol Ultrasound. 2018 May ; 59(3): 261-271. doi:10.1111/vru.12608.

\title{
Consensus recommendations on standardized magnetic resonance imaging protocols for multicenter canine brain tumor clinical trials
}

\author{
Rebecca A. Packer ${ }^{1}$, John H. RossmeisI ${ }^{2}$, Michael S. Kent ${ }^{3}$, John F. Griffin IV ${ }^{4}$, Christina \\ Mazcko $^{5}$, and Amy K. LeBlanc ${ }^{5}$ \\ ${ }^{1}$ Department of Clinical Sciences, College of Veterinary Medicine and Biomedical Sciences, \\ Colorado State University, Fort Collins, CO, 80523-1678 \\ ${ }^{2}$ Department of Small Animal Clinical Sciences, Virginia-Maryland College of Veterinary Medicine, \\ Blacksburg, VA, 24061 \\ ${ }^{3}$ Department of Surgical and Radiological Sciences, University of California Davis, School of \\ Veterinary Medicine, Davis, CA, 95616 \\ ${ }^{4}$ Department of Large Animal Clinical Sciences, College of Veterinary Medicine and Biomedical \\ Sciences, Texas A\&M University, College Station, TX, 77843 \\ ${ }^{5}$ Comparative Oncology Program, Center for Cancer Research, National Cancer Institute, \\ National Institutes of Health, Bethesda, MD, 20892
}

\begin{abstract}
The National Cancer Institute Comparative Brain Tumor Consortium, Patient Outcomes Working Group, propose a consensus document in support of standardized magnetic resonance imaging protocols for canine brain tumor clinical trials. The intent of this manuscript is to address the widely acknowledged need to ensure canine brain tumor imaging protocols are relevant and have sufficient equivalency to translate to human studies such that: (1) multi-institutional studies can be performed with minimal inter-institutional variation, and (2) imaging protocols are consistent with
\end{abstract}

Correspondence. Amy K LeBlanc, Comparative Oncology Program, Center for Cancer Research, National Cancer Institute, National Institutes of Health, 37 Convent Drive, Bethesda MD 20892, USA., amy.leblanc@nih.gov.

CONFLICT OF INTEREST

The authors declare they have no conflict of interest.

LIST OF AUTHOR CONTRIBUTIONS

Category 1
a. Conception and Design: LeBlanc AK, Packer RA, Mazcko C
b. Acquisition of Data: LeBlanc AK, Packer RA, Mazcko C, Griffin JF, Rossmeisl JH, Kent MS
c. Analysis and Interpretation of Data: LeBlanc AK, Packer RA, Mazcko C, Griffin JF, Rossmeisl JH, Kent MS

Category 2

a. Drafting the Article: LeBlanc AK, Packer RA, Mazcko C, Griffin JF, Rossmeisl JH, Kent MS

b. Revising Article for Intellectual Content: LeBlanc AK, Packer RA, Mazcko C, Griffin JF, Rossmeisl JH, Kent MS

Category 3

a. Final Approval of the Completed Article: LeBlanc AK, Packer RA, Mazcko C, Griffin JF, Rossmeisl JH, Kent MS 
human consensus recommendations to permit reliable translation of imaging data to human clinical trials. Consensus recommendations include pre- and postcontrast three-dimensional T1weighted images, $\mathrm{T} 2$-weighted turbo spin echo in all three planes, $\mathrm{T} 2 *$-weighted gradient recalled echo, T2-weighted fluid attenuated inversion recovery, and diffusion weighted imaging/diffusion tensor imaging in transverse plane; field of view of $₫ 50 \mathrm{~mm}$; slice thickness of $\mathcal{\Omega} \mathrm{mm}$, matrix $\geq$ 256 for two-dimensional images, and 150 or 256 for three-dimensional images.

\section{Keywords}

brain; clinical trials; consensus; magnetic resonance imaging; tumors

\section{1 | INTRODUCTION}

The National Cancer Institute's Comparative Brain Tumor Consortium was formed in September 2015, to collectively enhance cooperation among and collaboration between human and veterinary medical professionals and researchers involved in neurooncologic research. ${ }^{1}$ This Consortium's overarching mission is to improve the quality and forward progress of brain tumor research by leveraging the study of spontaneous brain tumors in pet dogs, and is an extension of the National Cancer Institute's Comparative Oncology Program, which has led the field of comparative oncology research for over a decade. There is substantial evidence that the study of naturally occurring cancers in dogs can meaningfully contribute to many fields of cancer research, including neurooncology, as tumor-bearing pet dogs are a disease model that complements knowledge gained in mouse models and through human clinical research. ${ }^{2-4}$ Within the Comparative Brain Tumor Consortium, five distinct working groups were formed to facilitate cross-discipline communications and generate new knowledge in the following areas: tumor biology, clinical trials, drug development, pathology and molecular markers, and patient outcomes. ${ }^{1}$

One specific goal of the Comparative Brain Tumor Consortium is to propose best practice guidelines for the conduct of multicenter clinical trials that evaluate novel drugs, devices, or imaging agents in canine brain tumor patients. These trials are designed to advance diagnostic or therapeutic strategies for humans, but also serve to enhance and expand our collective understanding of canine brain tumor biology. It is generally accepted that harmonized data capture protocols assure consistency and quality of the data generated therein, which is critical to increasing the likelihood of acceptance of comparative data by stakeholders in all facets of cancer research.

Members of the Comparative Brain Tumor Consortium patient outcomes working group proposed a consensus document in support of standardized magnetic resonance imaging (MRI) protocols for canine brain tumor clinical trials. This concept is derived from a similar consensus statement for human brain tumor trials. ${ }^{5}$ The proven benefit of using standardized imaging protocols has been realized in the Alzheimer's Disease Neuroimaging Initiative consensus as well as other initiatives. ${ }^{6,7}$ The intent of this manuscript is to offer initial consensus recommendations to ensure that multi-institutional veterinary brain tumor trials are robust and translationally relevant by employing standardized MRI protocols. The 
relative rarity of naturally occurring brain tumors in pet dogs necessitates cooperation among multiple veterinary centers that provide diagnostic and therapeutic avenues for pet dogs with brain tumors, including access to clinical trials.

Magnetic resonance imaging remains the imaging modality of choice for evaluating brain tumors. For both single and multi-institutional studies, adoption of guidelines for assessing response will allow the generation of robust data sets to evaluate the course of natural disease and response to interventions, while also supporting collection of data that are more comparable between studies. For these reasons, consensus on how to repeatedly and consistently image veterinary patients and evaluate tumor size, as well as other morphological and functional tumor evaluations, is essential.

Endpoints typically assessed in both human and veterinary clinical trials may include a variety of measures, yet all carry limitations in their ability to accurately portray the impact of a novel therapeutic strategy. For veterinary patients with brain tumors, overall survival, disease free interval, progression free survival, quality of life measures, and tumor responses can be difficult to measure and are potentially complicated by owners' discretionary resources and option of humane euthanasia in light of a brain tumor diagnosis. The assessment of tumor responses based on imaging data has become a standard surrogate for evaluating effectiveness of treatment in human neurooncology, and should be a useful tool in veterinary clinical studies as well. Indeed, MRI-based neuroimaging response criteria that describe various methods and associated challenges for measurement of tumor size and distinction from adjacent tissues have recently been described for canine brain tumor patients. ${ }^{8}$

Standardization of techniques used for capturing, reporting, and analysis of data gathered from cooperative groups is critical for maintaining quality of such findings. Specific to imaging trials, harmonization of image acquisition parameters has been specified as a key component of successful multi-site collaboration. ${ }^{9}$ These proposed consensus technique recommendations herein are not intended to be prescriptive, but rather provide a starting point from which minimum standards for consistent and functionally equivalent output can be achieved. It was also our intent to describe how these imaging standards could be applied throughout the conduct of a multicenter clinical trial that may evaluate a variety of therapeutic interventions. Indeed, harmonization standards for MRI-based characterization of epilepsy, based upon a parallel initiative in humans, have been provided to the veterinary community. ${ }^{10}$ Specific to oncology, standardized metrics for characterization and attribution of adverse events that occur during therapeutic trials, as well as for assessment of response to therapy in solid tumors and lymphomas, have been widely accepted and continue to play a key role in the acceptance of such data by the cancer drug development community. ${ }^{11-13}$

We believe that these proposed recommendations should be revisited regularly and revised accordingly as developments in technology, expertise, and knowledge occur. Successful application of the imaging guidelines proposed herein will guide and strengthen the validation and ongoing inclusion of the canine brain tumor model for human brain tumor research, and that these guidelines should continue to be modified and strengthened as 
knowledge and experience develops in the coming years, and as feedback is gathered and reviewed from veterinary instituitions whom choose to adopt these standards.

\section{2 | RECOMMENDED MAGNETIC RESONANCE IMAGING ACQUISITION AND OUTPUT PARAMETERS}

Minimum hardware requirements to achieve the recommended output parameters generally require 1.5T field strength or higher. In some cases, $<1.5 \mathrm{~T}$ systems may be utilized if the images are functionally equivalent in terms of output (e.g., voxel size, field-of-view, matrix, and signal-to-noise ratio); however, in most cases the longer scan times preclude feasibility of achieving these output parameters with lower field magnets. Regardless, the recommendations presented here are output-based to accommodate variations in hardware. Where vendor-specific sequence terminology is used, equivalent terminology and sequences may be available from other vendors.

Recommended sequence protocols should include, at a minimum, pre- and postcontrast three-dimensional T1-weighted images, T2-weighted turbo spin echo (TSE) in all three planes, T2*-weighted gradient recalled echo, T2-weighted fluid attenuated inversion recovery (FLAIR), and diffusion weighted imaging/diffusion tensor imaging in transverse plane (Table 1). The rationale for these sequences is described in more detail below, with consideration of relevant veterinary-specific MRI literature. ${ }^{14-18}$ To maximize scanning efficiency, recommendations as to the order of scanning are presented in Table 1 and described later in the text.

\section{1 | Output parameters}

A field of view of $\$ 50 \mathrm{~mm}$ is recommended in all three planes for both two-dimensional and three-dimensional images. Three-dimensional T1-weighted images should have isotropic voxels $\leq \mathrm{mm}$, with no gap and no overlap. Slice thickness should be $\_\mathrm{mm}$ for two-dimensional brain images, with $2 \mathrm{~mm}$ considered ideal. Matrix size should be $\geq 256$ for two-dimensional brain images. Provided the field of view is $150 \mathrm{~mm}$, these parameters would create voxels that are no larger than $0.58 \times 0.58 \times 3 \mathrm{~mm}$ for two-dimensional images, and either $0.6 \mathrm{~mm}$ (if matrix is $256 \mathrm{~mm}$ ) or $1 \mathrm{~mm}$ (if matrix is $150 \mathrm{~mm}$ ) isotropic voxels for three-dimensional images.

The ideal MRI slice thickness in dogs has not been determined, although it is thought that thinner slices with no spacing allows for better tumor delineation. Since the canine brain is smaller than the human brain, the recommended minimum MRI slice thickness in dogs is thinner than recommended for humans. In human patients, the Response Assessment in Neuro-Oncology criteria recommend a minimum MRI slice thickness of $5 \mathrm{~mm}$ with no interslice spacing for evaluation. ${ }^{19}$ In order to reduce volume averaging, a slice thickness of $\widehat{\imath} \mathrm{mm}$ is recommended as ideal for canine subjects.

\section{2 | Effect of field strength}

In general, higher field strength results in improved spatial resolution, contrast-to-noise ratio, and signal-to-noise ratio relative to scan time. ${ }^{20-22}$ Theoretically, this should result in 
improved lesion margin delineation and conspicuity. That being said, rigorous studies comparing field strength within canine subjects with brain tumors have not been performed, and studies in human subjects with brain tumors focused largely on contrast enhancement relative to field strength, rather than noncontrast sequences. ${ }^{20-22}$ When planning multicenter studies involving different field strengths, it is important that certain differences are considered, aside from tumor delineation. For example, certain artifacts, such as chemical shift and magnetic susceptibility artifact, can be more problematic at higher fields, such as $3 T^{23,24}$ Although compensation can be achieved by increasing bandwidth, this in turn affects signal-to-noise ratios. Such differences as a result of field strength and limitations must be considered in multi-institutional trials.

\section{3 | T2-weighted sequences}

T2-weighted TSE sequences should be acquired in all three planes. T2-weighted sagittal and dorsal images are generally acquired earlier in the scanning protocol to permit rapid assessment of anatomic structures. Similar to the human consensus recommendations, T2weighted transverse sequences may be acquired after gadolinium administration to standardize the timing of postcontrast T1-weighted image acquisition and to maximize efficiency by actively scanning during the delay after contrast administration. Although gadolinium can effect T2-weighted imaging, the effects are minimal at clinically relevant gadolinium concentrations and time of echo. As such, for T2-weighted images, performing the T2-weighted acquisition after gadolinium administration should have no effect on image integrity.

\section{4 | T2-weighted flame-attenuated inversion recovery sequences}

T2-weighted FLAIR sequences should be acquired as part of the minimum acquisition for brain tumor clinical trials. The T2-weighted FLAIR sequence is recommended primarily to aid in lesion assessment adjacent to cerebral spinal fluid-filled structures. Importantly, many canine gliomas share a margin with the ventricles and are difficult to fully delineate on T2weighted images made without inversion recovery. ${ }^{25}$

\section{5 | T2*-weighted sequences}

Even though the human recommendations do not include a T2*-weighted sequence, we chose to include this in the canine recommendations for two reasons. First, imaging findings in dogs with hemorrhagic infarcts can overlap with those of neoplasia. ${ }^{17,26}$ In longitudinal studies, it may be important to recognize changes in lesion size caused by intratumoral or peritumoral hemorrhage that may be related to interventions included within the clinical trial schema.

\section{6 | Three-dimensional T1-weighted sequences}

When possible, the centers participating in the clinical trial should perform threedimensional T1-weighted sequences that are similar with respect to pulse sequence type (gradient echo or spin echo), field-of-view, and image contrast. ${ }^{27-30} \mathrm{We}$ recommend chemical fat saturation in order to improve sensitivity for the detection of gadolinium contrast medium. ${ }^{31}$ There are several advantages of three-dimensional sequences over 
standard two-dimensional sequences. For example, images acquired in three dimensions can be reconstructed in the transverse, sagittal, and dorsal plane with reduced acquisition time compared to acquiring two-dimensional images in all three planes. Furthermore, tumor volume estimation utilizing three-dimensional sequences is more straightforward because of the isotropic voxels, lack of interslice gaps, and reduced partial volume effects. These observations are reiterated in published human and veterinary literature, specifically with respect to the significant variability in one- and two-dimensional measurements due in large part to variation in slice location between serial scans. ${ }^{32-34}$

While two-dimensional spin echo imaging may produce images with increased conspicuity of contrast medium, three-dimensional imaging performs similarly in lesion detection and may depict nonenhancing tumors better. ${ }^{35,36}$ Comparing three-dimensional TSE and gradient echo sequences at 3T, the TSE images have the advantage of reduced vascular signals (which could mimic neoplasia) while gradient echo images have superior gray/white matter distinction. ${ }^{37}$

\section{7 | Diffusion-weighted sequences}

Diffusion weighted imaging or diffusion tensor imaging is recommended for several reasons. First, may aid in the differentiation of dogs with neoplasia from those with ischemic infarcts.

${ }^{38}$ Second, it provides quantitative metrics (such as apparent diffusion coefficient and fractional anisotropy) which can be tracked longitudinally. Third, there is evidence that diffusion imaging may aid in differentiation of post-radiation changes (pseudoprogression) from tumor progression. ${ }^{39-44}$ SS-EPI sequences have the advantage of short acquisition times; however, single shot echo planar imaging diffusion sequences often result in distortion of the olfactory region due to susceptibility artifact from the frontal sinuses, particularly at 3T. At 3T, the use of multi-shot echo planar imaging sequences or spin echo sequences (such as the BLADE sequence) may reduce this artifact. It is important to note that this is one instance where differences in canine and human anatomy affect what otherwise would be recommended in human consensus imaging protocols. Diffusion images should be acquired at $b=0, b=1000$ (or maximum per system), and where possible to improve precision, an intermediate $b$-value.

\section{8 | Contrast imaging}

Dosing and timing of contrast-medium administration prior to image acquisition should be standardized for the study protocol as well as across multiple sites. Normal contrast enhancement of the choroid plexus, meningeal blood vessels and nasal mucosa should be readily apparent on each study. Where possible, the use of subtraction images to evaluate contrast-enhancement is recommended. ${ }^{45-49}$ Performing comparable T1-weighted sequences both pre- and postcontrast permits the use of subtraction images to evaluate contrast enhancement, which can be performed automatically on most proprietary and open source commercial image analysis platforms and does not require sequential imaging. However, if pre- and post-gadolinium images are acquired sequentially as a dynamic sequence, T2-weighted images cannot be acquired in the interim. ${ }^{49}$ 
There is evidence in the literature that subtraction scans are superior for monitoring tumor progression. ${ }^{48}$ The optimal duration between contrast medium injection and imaging is not known. That being said, a delay of at least 5 min seems to be beneficial and prolonged delays have not resulted in improved characterization of meningeal enhancement in dogs. 31,47

\section{9 | Image processing and reporting}

All studies must be DICOM-send compliant to permit multi-institutional transfer for image review and analysis. Clinical trial centers should consider whether a central read at a designated site and/or with a blinded review is required to avoid interobserver variation or bias. Where a central read is not standard for a multi-institutional trial, minimum standards for reporting elements should be shared across all participating sites.

\section{3 | GUIDELINES ON IMPLEMENTATION OF STANDARDIZED MAGNETIC RESONANCE IMAGING PROTOCOLS}

\section{1 | High-level considerations and compromises}

This manuscript is meant to provide a framework for brain tumor clinical trials that include MRI to support the consistent, clear, and reproducible methodology that should be considered the minimum standard for conductance of such comparative canine trials moving forward. While we collectively acknowledge the variability in hardware and software between sites, we similarly acknowledge the need for prospective determination of imaging protocol prior to initiation of the trial among the participating investigators. We envision a pragmatic approach wherein a realistic balance can be struck between an ideal scenario involving high-performance systems that may not be widely available even among veterinary academic institutions, and what can be reasonably achieved while maintaining and promoting compliance and acceptance from the collaborating parties and the larger comparative brain tumor research community. As the comparative clinical study of canine brain tumors as models for humans develops, there is a clear responsibility for veterinarians involved in directing/executing the clinical trials to maintain the highest standards possible for imaging endpoints, which are critical for the monitoring of veterinary patients enrolled in interventional studies.

The minimal sequences obtained for evaluation of tumor size should include pre- and postcontrast three-dimensional T1-weighted images (which can be reconstructed in all three planes), T2-weighted TSE in all three planes, T2*-weighted gradient recalled echo, T2weighted FLAIR, and diffusion weighted imaging/diffusion tensor imaging in transverse plane. ${ }^{5}$ Additional sequences should be done based on the clinical and study needs, but should be prospectively determined for each study. Imaging sequences that provide functional and metabolic information about a tumor can also be done (such as MRI spectroscopy, proton spectroscopy, and perfusion weighted imaging), although at this point these are still not developed enough for use in evaluating responses on their own.

In human neurooncology practice, several systems have been used to assess brain tumor responses. Criteria for high grade gliomas include the RECIST criteria, the Macdonald 
method, and the Response Assessment in Neuro-Oncology criteria. ${ }^{19,50,51}$ There are additional published Response Assessment in Neuro-Oncology criteria for diffused low grade glial tumors, pediatric high grade gliomas, brain metastasis, and spinal metastasis, as well as specific response assessments guidelines for immunotherapeutic trials. ${ }^{5,52-55}$ Criteria for meningioma, despite it being one of the more common brain tumor histologies seen, are not yet available, although they are being developed. ${ }^{56}$ Separate criteria have also been developed for immunotherapy and surgical trials in neurooncology. ${ }^{55,57}$ All three of these methods involve one- or two-dimensional diameter-based measurements with the Macdonald and Response Assessment in Neuro-Oncology criteria also taking into account clinical response including the dependence on steroid use. For high grade gliomas the Response Assessment in Neuro-Oncology criteria also subjectively takes into account the T2 or FLAIR area in assessing response. Volumetric assessment methods are yet to be standardized and include using varying software packages. ${ }^{58}$

There is some information in the veterinary literature on the assessment of serial images to determine brain tumor treatment response and no widely agreed-upon methods have been published to date, although several studies have used published human criteria. ${ }^{8,59}$ The reader is directed to a previous publication that compares and contrasts RECIST, Macdonald, Response Assessment in Neuro-Oncology, and volumetric methods (Table 3). ${ }^{8}$ At the current time, adoption of the human Response Assessment in Neuro-Oncology criteria, can be readily applied to other tumor types and should be considered. Alternatively, other three-dimensional volumetric methods can be used in addition with appropriate reporting of the imaging and methodology used so they can be repeated by other groups until such time as there is consensus on the use of these methods. Nonetheless, limitations remain related to accuracy of imaging (in particular contrast-enhancement) for assessments of tumor volume or growth. Although some evidence exists to guide assessment of pseudoprogression vs. progression, as of yet there is no widely accepted ideal.

\section{2 | Interobserver variability}

To limit measurement variability within a study the same DICOM viewing software and the same observer should evaluate each image set within a study even for multi-institutional studies. The use of multiple observers taking the same measurement independently to limit and assess interobserver effects should be considered.

\section{3 | Baseline imaging prior to enrollment into a clinical trial}

To avoid significant alterations in the tumor or peritumoral environments between the time of imaging diagnosis and any intended intervention, baseline MRI examinations should be performed in close proximity to the time of trial enrollment. In human clinical trials, inclusion criteria often dictate that baseline MRI examinations be obtained within 2 weeks of enrollment in studies investigating primary high-grade neuroepithelial and metastatic tumors, whereas slightly longer intervals of 4-8 weeks may be acceptable for slower growing tumors such as meningiomas. ${ }^{60}$ Baseline MRI examinations should be obtained while the patient's neurological status and corticosteroid dose are stable, to avoid variation in apparent tumor volume or clinical response simply from the influence of corticosteroids (apparent decrease in tumor volume and clinical signs) or seizures (apparent increase in 
tumor volume). Previous investigations have demonstrated that seizures can induce transient magnetic resonance signal alterations in the brain, and corticosteroid therapy can reduce both the peritumoral edema and tumor burdens. ${ }^{61-64}$ Baseline MRI datasets should also contain identical sequences and image planes to those that will be obtained for analysis following an intervention.

\section{4 | Serial imaging}

Serial imaging should be done using the same pulse sequence parameters, slice thickness, the same dose and type of contrast medium, and the same duration between contrast medium administration and imaging. Consistency in these factors is important, as minor differences in protocol parameters or hardware result in significant differences in image output, as described elsewhere in this text.

For now, adoption of the human Response Assessment in Neuro-Oncology criteria, which can be readily applied to other tumor types, should be considered. Alternatively, other threedimensional volumetric methods can be used in addition with appropriate reporting of the imaging and methodology used so they can be repeated by other groups until such time as there is consensus on the use of these methods.

\section{5 | Timing of magnetic resonance imaging}

Given the current knowledge gaps regarding the natural history of disease and the lack of evidence-based, standard-of-care therapies for spontaneous brain tumors in companion animals, the ideal intervals for the performance of MRI surveillance are largely unknown. 65,66 The human neurooncology experience with development and incorporation of imagingbased therapeutic response assessments into brain clinical trials has demonstrated that all aspects of the process require continual adaptation and evolution in response to new discoveries and technologies. ${ }^{67,68}$ Thus, while the optimization of the timing of posttreatment imaging in veterinary medicine will be dependent on factors including tumor histology and grade, the anticipated therapeutic and adverse effects of the treatment administered, the patient's clinical status, and the defined end-points of each clinical trial, these recommendations should remain subject to change as our understanding of brain tumors progresses. Additional considerations include expected responses and outcomes to limit loss of data points, and treatment interventions that may result in either pseudoresponses or pseudoprogression. In animals with spontaneous brain tumors, an additional practical consideration regarding the scheduled frequency of posttreatment MRI surveillance is the necessity to anesthetize patients in order to obtain diagnostic datasets. $8,65,66$

There are several issues that confound extraction of evidenced-based recommendations from the literature regarding the timing of posttreatment MRI for canine brain tumors. The majority of studies investigating therapeutic endpoints in large numbers of dogs with brain tumors are retrospective, contain populations with heterogeneous and often presumptively diagnosed tumor types or that received variable treatments, and do not incorporate imagebased response assessments into outcome analyses. ${ }^{69-72}$ Currently, very few reports exist in dogs with naturally occurring brain tumors in which study designs incorporated a specific 
tumor histology (i.e., glioma) and serial posttreatment imaging examinations performed at standardized intervals. ${ }^{73-75}$ These reports have limitations such as treatment of presumptively diagnosed tumors, inclusion of small cohorts of dogs, and performance of novel treatments that preclude generalized extrapolation of the timing of imaging surveillance to similar dog populations treated with conventional modalities, but provide a reasonable framework upon which future protocols can be modeled. The results also highlight the feasibility and need to incorporate imaging-based endpoints into translational canine brain tumor trials by demonstrating the value of and challenges associated with MRI for the detection, characterization, and discrimination of tumor progression, treatmentrelated effects, and toxicities..$^{8,73-75}$

In canine clinical trials, in which the effects of investigational therapeutics on brain tumors are frequently unknown, initial followup MRI examinations have been performed at 6 weeks following treatment. Examples of such trial designs currently in use in dogs with brain tumors can be found here: https://ebusiness.avma.org/aahsd/study_search_results.aspx. This interval approximates the observed $80 \%$ survival rate of dogs with primary brain tumors treated palliatively, and thus minimizes attrition due to death or euthanasia prior to obtaining posttreatment imaging. ${ }^{8,70}$ Accounting for these factors and complexities, Table 2 summarizes the recommendations for timing of imaging for $3 \mathrm{~T}$ and $<3 \mathrm{~T}$ MRI units.

\section{6 | Early postoperative imaging}

In humans, surgery is a primary therapeutic modality for brain tumors, and is often the only treatment used for low-grade tumors. Given the established and emerging prognostic importance of the extent of resection for many human brain tumors, early post-operative MRI has emerged as the preferred method to objectively evaluate extent of resection. ${ }^{76-78}$ Early postoperative MRI evaluations play an important role in postsurgical patient management and subsequent monitoring of the effects of adjuvant therapies, and are crucial for clinical trials with qualitative (subtotal versus complete resection) or quantitative (volumetric reduction threshold) extent of resection end-points. Surgery has also been demonstrated to be an effective modality for the treatment of canine and feline meningiomas and functional pituitary tumors, although no studies have reported associations between imaging derived measures of the extent of resection and patient outcomes. ${ }^{65,79-81}$

Surgical intervention in both human and veterinary patients poses unique challenges to the interpretation of imaging-based therapeutic response assessments performed in the acute postoperative period. Sequelae of surgical manipulation can include the presence of air, edema, hemorrhage, hemostatic agents, ischemia, reactive contrast-enhancement, and susceptibility artifacts from metallic surgical instrumentation within the operative field that compromise the evaluation of complications or residual tumor. ${ }^{8,82-84}$ To avoid difficulties with differentiation of benign contrast-enhancement from residual contrast-enhancing tumor, it has been recommended that early postoperative MRI examinations obtained on 1.5T units be performed within $72 \mathrm{~h}$ of surgery, as prior studies have demonstrated that reactive enhancement does not develop within $72 \mathrm{~h}$ of surgery. ${ }^{85}$ However, the MRI field strength needs to be a factor when optimizing timing of early postoperative imaging, as the degree of contrast-enhancement of brain tumors is dependent on magnetic field strength. ${ }^{86-88}$ With the 
more widespread use of 3T MRI units in the clinical setting, reactive contrast-enhancement may be detected in a significant number of patients imaged within this previously recommended $72 \mathrm{~h}$ early postoperative window, and may be observed as early as $24 \mathrm{~h}$ postoperatively. ${ }^{89}$ Discrimination between the etiologies of postoperative contrastenhancement may also be facilitated by assessment of the location, pattern, and margination of early postoperative contrast enhancement. Reactive-enhancement typically appears as fine linear regions of enhancement conforming to the margins of the resection bed or regional meninges. ${ }^{83,84}$

Studies describing posttreatment imaging following radiotherapeutic treatment of veterinary brain tumors indicate typical intervals of 3-6 months, or reported methods do not describe adhence to a standardized interval. ${ }^{59,71-73}$ However, many of these studies consist of heterogeneous patient populations that include multiple types of presumptively diagnosed brain tumors, which complicates formulation of recommendations regarding specific followup intervals. Differentiation of treatment induced changes from tumor progression on posttreatment MRI examinations remains a challenge. ${ }^{8,76}$

\section{7 | Adjunctive patient outcome assessments}

Postmortem evaluation of all deceased subjects should be included as a goal in all prospective veterinary trials to allow for assessment of histology, tumor response, and any associated toxicity. This is especially important in veterinary neurooncology studies where preintervention histopathology may not be available and the tumor diagnosis may be based on imaging criteria.

\section{4 | CONCLUSIONS}

In summary, authors of this commentary review believe that prospectively defining image acquisition and reporting criteria is essential to maximize the value of veterinary neurooncology studies, and that these guidelines reflect a starting point for minimum standards that could lead to greater cooperation among the brain tumor research community and acceptance of canine patient as a translationally relevant model for humans. Where available, the human Response Assessment in Neuro-Oncology criteria based on the tumor histology being studied should be applied as a response criteria in veterinary studies. Until such time that the Response Assessment in Neuro-Oncology criteria for meningiomas is developed, the Response Assessment in Neuro-Oncology criteria for high grade gliomas can be used to evaluate a response to therapy. The response and reporting criteria being recommended in this document are designed to limit variability both within and between studies and provide more robust data for veterinary observational and clinical trials. This is proposed as a working document. These guidelines should continue to be modified and strengthened as evidence-based knowledge and experience develop in the coming years, and be revised accordingly as developments in technology, expertise, and knowledge occur.

As a number of equipment vendors exist currently, and as we cannot foresee what imaging technology will be emerging in the future, the consensus recommendations contained in this paper represent minimum output standards to permit functional equivalency for sites participating in multicenter canine brain tumor clinical trials. These recommendations are 
not meant to limit those sites that wish to surpass these minimum standards. The intended use of these recommendations is simply to support multiinstitutional collaborations and more relevant comparisons to other studies. Further, it is worth considering adopting these as minimum standards in any patient with an intracranial localization, such that if a brain tumor is found, the images would permit post-hoc entry into a clinical trial.

\section{Acknowledgments}

Members of the National Cancer Institute Comparative Brain Tumor Consortium (NCI CBTC) Patient Outcomes Working Group: Tim Bentley, Purdue University Veterinary Medicine; Annie Chen-Allen, Washington State University College of Veterinary Medicine; Mark Gilbert, National Cancer Institute; Jonathan Levine, Texas A\&M University; Laura Selmic, University of Illinois; Amanda Taylor, Auburn University; Evelyn Galban, University of Pennsylvania; Jing Wu, National Cancer Institute.

\section{Funding statement}

This research was supported in part by the Intramural Research Program of the National Institutes of Health (NIH), NCI, Center for Cancer Research. The results and interpretations do not reflect the views of the US Government.

\section{References}

1. LeBlanc AK, Mazcko C, Brown DE, et al. Creation of an NCI comparative brain tumor consortium: informing the translation of new knowledge from canine to human brain tumor patients. Neuro Oncol. 2016; 18:1209-1218. [PubMed: 27179361]

2. Stroud C, Dmitriev I, Kashentseva E, et al. A One Health overview, facilitating advances in comparative medicine and translational research. Clin Transl Med. 2016; 5(Suppl 1):26. [PubMed: 27558513]

3. LeBlanc AK, Mazcko CN, Khanna C. Defining the value of a comparative approach to cancer drug development. Clin Cancer Res. 2016; 22:2133-2138. [PubMed: 26712689]

4. LeBlanc AK, Breen M, Choyke P, et al. Perspectives from man's best friend: National Academy of Medicine's workshop on comparative oncology. Sci Transl Med. 2016; 8:324ps325.

5. Ellingson BM, Bendszus M, Boxerman J, et al. Consensus recommendations for a standardized brain tumor imaging protocol in clinical trials. Neuro Oncol. 2015; 17:1188-1198. [PubMed: 26250565]

6. Weiner MW, Veitch DP, Aisen PS, et al. Impact of the Alzheimer's disease neuroimaging initiative, 2004 to 2014. Alzheimers Dement. 2015; 11:865-884. [PubMed: 26194320]

7. Mueller SG, Weiner MW, Thal LJ, et al. The Alzheimer's disease neuroimaging initiative. Neuroimaging Clin N Am. 2005; 15:869-877. [PubMed: 16443497]

8. Rossmeisl JH Jr, Garcia PA, Daniel GB, et al. Invited review-Neuroimaging response assessment criteria for brain tumors in veterinary patients. Vet Radiol Ultrasound. 2014; 55:115-132. [PubMed: 24219161]

9. Clarke LP, Nordstrom RJ, Zhang H, et al. The quantitative imaging network: NCI's historical perspective and planned goals. Transl Oncol. 2014; 7:1-4. [PubMed: 24772201]

10. Rusbridge C, Long S, Jovanovik J, et al. International veterinary epilepsy task force recommendations for a veterinary epilepsy-specific MRI protocol. BMC Vet Res. 2015; 11:194. [PubMed: 26319136]

11. Anonymous. Veterinary cooperative oncology group. Common terminology criteria for adverse events (VCOG-CTCAE) following chemotherapy or biological antineoplastic therapy in dogs and cats v1.1. Vet Comp Oncol. 2016; 14:417-446. [PubMed: 28530307]

12. Nguyen SM, Thamm DH, Vail DM, London CA. Response evaluation criteria for solid tumours in dogs (v1.0): A Veterinary Cooperative Oncology Group (VCOG) consensus document. Vet Comp Oncol. 2015; 13:176-183. [PubMed: 23534501]

13. Vail DM, Michels GM, Khanna C, Selting KA, London CA. Veterinary Cooperative Oncology Group. Response evaluation criteria for peripheral nodal lymphoma in dogs (v1.0) - A Veterinary 
Cooperative Oncology Group (VCOG) consensus document. Vet Comp Oncol. 2010; 8:28-37. [PubMed: 20230579]

14. Gross B, Garcia-Tapia D, Riedesel E, Ellinwood NM, Jens JK. Normal canine brain maturation at magnetic resonance imaging. Vet Radiol Ultrasound. 2010; 51:361-373. [PubMed: 20806866]

15. Hodshon AW, Hecht S, Thomas WB. Use of the T2*-weighted gradient recalled echo sequence for magnetic resonance imaging of the canine and feline brain. Vet Radiol Ultrasound. 2014; 55:599606. [PubMed: 24833062]

16. Hartmann A, Söffler C, Failing K, Schaubmar A, Kramer M, Schmidt MJ. Diffusion-weighted Magnetic Resonance Imaging of the normal canine brain. Vet Radiol Ultrasound. 2014; 55:592598. [PubMed: 24798796]

17. Young BD, Mankin JM, Griffin JF, Fosgate GT, Fowler JL, Levine JM. Comparison of two fatsuppressed magnetic resonance imaging pulse sequences to standard T2-weighted images for brain parenchymal contrast and lesion detection in dogs with inflammatory intracranial disease. Vet Radiol Ultrasound. 2015; 56:204-211. [PubMed: 25395066]

18. Wolff CA, Holmes SP, Young BD, et al. Magnetic Resonance Imaging for the differentiation of neoplastic, inflammatory, and cerebrovascular brain disease in dogs. J Vet Intern Med. 2012; 26:589-597. [PubMed: 22404482]

19. Wen PY, Chang SM, Van den Bent MJ, et al. Response assessment in neuro-oncology clinical trials. J Clin Oncol. 2017; 35:2439-2449. [PubMed: 28640707]

20. Krautmacher C, Willinek WA, Tschampa HJ, et al. Brain tumors: Full-and half-dose contrastenhanced MR imaging at 3.0 T compared with 1.5 T-Initial Experience. Radiology. 2005; 237:1014-1019. [PubMed: 16237142]

21. Ba-Ssalamah A, Nobauer-Huhmann IM, Pinker K, et al. Effect of contrast dose and field strength in the magnetic resonance detection of brain metastases. Invest Radiol. 2003; 38:415-422. [PubMed: 12821855]

22. Chang KH, Ra DG, Han MH, Cha SH, Kim HD, Han MC. Contrast enhancement of brain tumors at different MR field strengths: comparison of 0.5 T and 2.0 T. AJNR Am J Neuroradiol. 1994; 15:1413-1419. [PubMed: 7985558]

23. Soher BJ, Dale BM, Merkle EM. A review of MR physics: 3T versus 1.5T. Magn Reson Imaging Clin N Am. 2007; 15:277-290. [PubMed: 17893049]

24. Farahani K, Sinha U, Sinha S, Chiu LC, Lufkin RB. Effect of field strength on susceptibility artifacts in magnetic resonance imaging. Comput Med Imaging Graph. 1990; 14:409-413. [PubMed: 2272012]

25. Young BD, Levine JM, Porter BF, et al. Magnetic resonance imaging features of intracranial astrocytomas and oligodendrogliomas in dogs. Vet Radiol Ultrasound. 2011; 52:132-141. [PubMed: 21388463]

26. Young BD, Fosgate GT, Holmes SP, et al. Evaluation of standard magnetic resonance characteristics used to differentiate neoplastic, inflammatory, and vascular brain lesions in dogs. Vet Radiol Ultrasound. 2014; 55:399-406. [PubMed: 24467341]

27. Ellingson BM, Bendszus M, Sorensen AG, Pope WB. Emerging techniques and technologies in brain tumor imaging. Neuro Oncol. 2014; 16(Suppl 7):vii12-23. [PubMed: 25313234]

28. Glaser C, D'Anastasi M, Theisen D, et al. Advantages, disadvantages, and application in MSK imaging. Semin Musculoskelet Radiol. 2015; 19:321-327. [PubMed: 26583360]

29. Fan Z, Yang Q, Deng Z, et al. Whole-brain intracranial vessel wall imaging at 3 Tesla using cerebrospinal fluid-attenuated T1-weighted 3D turbo spin echo. Magn Reson Med. 2017; 77:1142-1150. [PubMed: 26923198]

30. Lee S, Park DW, Lee JY, Lee YJ, Kim T. Improved motion-sensitized driven-equilibrium preparation for 3D turbo spin echo T1 weighted imaging after gadolinium administration for the detection of brain metastases on 3T MRI. Br J Radiol. 2016; 89:20150176. [PubMed: 27187597]

31. D'Anjou MA, Carmel EN, Blond L, Beauchamp G, Parent J. Effect of acquisition time and chemical fat suppression on meningeal enhancement on MR imaging in dogs. Vet Radiol Ultrasound. 2012; 53:11-20. [PubMed: 22092922] 
32. Stadler KL, Ruth JD, Pancotto TE, et al. Computed tomography and magnetic resonance imaging are equivalent in mensuration and similarly inaccurate in type and grade predictability of canine intracranial gliomas. Front Vet Sci. 2017; 4:157. [PubMed: 28993810]

33. Wang MY, Cheng JL, Han YH, et al. Comparison of volumetric methods for tumor measurements on two and three dimensional MRI in adult glioblastoma. Neuroradiology. 2011; 53:565-569. [PubMed: 21057780]

34. Kanaly CW, Ding D, Mehta AI, et al. A novel method for volumetric MRI response assessment of enhancing brain tumors. PLoS One. 2011; 6:e16031. [PubMed: 21298088]

35. Dodo T, Okada T, Yamamoto A, et al. T1-weighted MR imaging of glioma at 3T: A comparative study of 3D MPRAGE vs. conventional 2D spin-echo imaging. Clin Imaging. 2016; 40:12571261. [PubMed: 27639863]

36. Zhu W, Qi J, Wang C. Comparative study of 3D-SPGR vs 2D-SE T1WI after enhancement in the brain. J Huazhong Univ Sci Technol Med Sci. 2003; 23:180-183. [PubMed: 12973944]

37. Komada T, Naganawa S, Ogawa H, et al. Contrast-enhanced MR imaging of metastatic brain tumor at 3 Tesla: utility of T(1)-weighted SPACE compared with 2D spin echo and 3D gradient echo sequence. Magn Reson Med Sci. 2008; 7:13-21. [PubMed: 18460844]

38. Sutherland-Smith J, King R, Faissler D, Ruthazer R, Sato A. Magnetic resonance imaging apparent diffusion coefficients for histologically confirmed intracranial lesions in dogs. Vet Radiol Ultrasound. 2011; 52:142-148. [PubMed: 21388464]

39. Prager AJ, Martinez N, Beal K, Omuro A, Zhang Z, Young RJ. Diffusion and perfusion MRI to differentiate treatment-related changes including pseudoprogression from recurrent tumors in highgrade gliomas with histopathologic evidence. AJNR Am J Neuroradiol. 2015; 36:877-885. [PubMed: 25593202]

40. Matsusue E, Fink JR, Rockhill JK, Ogawa T, Maravilla KR. Distinction between glioma progression and post-radiation change by combined physiologic MR imaging. Neuroradiology. 2010; 52:297-306. [PubMed: 19834699]

41. Galban S, Brisset JC, Rehemtulla A, Chenevert TL, Ross BD, Galban CJ. Diffusion-weighted MRI for assessment of early cancer treatment response. Curr Pharm Biotechnol. 2010; 11:701-708. [PubMed: 20504274]

42. Padhani AR, Khan AA. Diffusion-weighted (DW)and dynamic contrast-enhanced (DCE) magnetic resonance imaging (MRI) for monitoring anticancer therapy. Target Oncol. 2010; 5:39-52. [PubMed: 20383784]

43. Padhani AR, Liu G, Koh DM, Chenevert TL, et al. Diffusion-weighted magnetic resonance imaging as a cancer biomarker: consensus and recommendations. Neoplasia. 2009; 11:102-125. [PubMed: 19186405]

44. Erickson BJ, Wood CP, Kaufmann TJ, Patriarche JW, Mandrekar J. Optimal presentation modes for detecting brain tumor progression. AJNR Am J Neuroradiol. 2011; 32:1652-1657. [PubMed: 21852368]

45. Dirrig H, Lamb CR. Magnetic resonance imaging of intracranial inflammatory conditions in dogs: Sensitivity of subtraction images versus pre- and post-gadolinium T1-weighted image pairs. Vet Radiol Ultrasound. 2016; 57:410-416. [PubMed: 27144775]

46. Lee VS, Flyer MA, Weinreb JC, Krinsky GA, Rofsky NM. Image subtraction in gadoliniumenhanced MR imaging. AJR Am J Roentgenol. 1996; 167:1427-1432. [PubMed: 8956572]

47. Joslyn S, Sullivan M, Novellas R, Brennan N, Cameron G, Hammond G. Effect of delayed acquisition times on gadolinium-enhanced magnetic resonance imaging of the presumably normal canine brain. Vet Radiol Ultrasound. 2011; 52:611-618. [PubMed: 21831250]

48. Melhem ER, Mehta NR. Dynamic T1-weighted spin-echo MR imaging: the role of digital subtraction in the demonstration of enhancing brain lesions. J Magn Reson Imaging. 1999; 9:503508. [PubMed: 10232507]

49. Lamb CR, Lam R, Keenihan EK, Frean S. Appearance of the canine meninges in subtraction magnetic resonance images. Vet Radiol Ultrasound. 2014; 55:607-613. [PubMed: 24833219]

50. Wen PY, Macdonald DR, Reardon DA, et al. Updated response assessment criteria for high-grade gliomas: response assessment in neuro-oncology working group. J Clin Oncol. 2010; 28:19631972. [PubMed: 20231676] 
51. Macdonald DR, Cascino TL, Schold SC Jr, Cairncross JG. Response criteria for phase II studies of supratentorial malignant glioma. J Clin Oncol. 1990; 8:1277-1280. [PubMed: 2358840]

52. van den Bent MJ, Wefel JS, Schiff D, et al. Response assessment in neuro-oncology (a report of the RANO group): Assessment of outcome in trials of diffuse low-grade gliomas. Lancet Oncol. 2011; 12:583-593. [PubMed: 21474379]

53. Jaspan T, Morgan PS, Warmuth-Metz M, et al. Response assessment in pediatric neuro-oncology: Implementation and expansion of the RANO criteria in a randomized phase II trial of pediatric patients with newly diagnosed high-grade gliomas. AJNR Am J Neuroradiol. 2016; 37:1581-1587. [PubMed: 27127006]

54. Lin NU, Lee EQ, Aoyama H, et al. Response assessment criteria for brain metastases: Proposal from the RANO group. Lancet Oncol. 2015; 16:e270-8. [PubMed: 26065612]

55. Okada H, Weller M, Huang R, et al. Immunotherapy response assessment in neuro-oncology: A report of the RANO working group. Lancet Oncol. 2015; 16:e534-42. [PubMed: 26545842]

56. Rogers CL, Perry A, Pugh S, et al. Pathology concordance levels for meningioma classification and grading in NRG oncology RTOG trial 0539. Neuro Oncol. 2016; 18:565-574. [PubMed: 26493095]

57. Vogelbaum MA, Jost S, Aghi MK, et al. Application of novel response/progression measures for surgically delivered therapies for gliomas: Response assessment in neuro-oncology (RANO) working group. Neurosurgery. 2012; 70:234-243. discussion 243-4. [PubMed: 21593697]

58. Kanaly CW, Mehta AI, Ding D, et al. A novel, reproducible, and objective method for volumetric magnetic resonance imaging assessment of enhancing glioblastoma. J Neurosurg. 2014; 121:536542. [PubMed: 25036205]

59. Zwingenberger AL, Pollard RE, Taylor SL, Chen RX, Nunley J, Kent MS. Perfusion and volume response of canine brain tumors to stereotactic radiosurgery and radiotherapy. J Vet Intern Med. 2016; 30:827-835. [PubMed: 27149650]

60. Henson JW, Ulmer S, Harris GJ. Brain tumor imaging in clinical trials. Am J Neuroradiol. 2008; 29:419-424. [PubMed: 18272557]

61. Cianfoni A, Caulo M, Cerase G, Della Marca C, Falcone Gm, Di Lella S, Gaudino J, Colosimo E. Seizure-induced brain lesions: A wide spectrum of variably reversible MRI abnormalities. Eur J Radiol. 2013; 82:1964-1972. [PubMed: 23787273]

62. Ong B, Bergin P, Heffernan T, Stuckey S. Transient seizure-related MRI abnormalities. J Neuroimaging. 2009; 19:301-310. [PubMed: 19226345]

63. Mellema LM, Koblik PD, Kortz GD, LeCouteur RA, Chechowitz MA, Dickinson PJ. Reversible magnetic resonance imaging abnormalities in dogs following seizures. Vet Rad Ultrasound. 1999; 40:588-595.

64. Smith PM, Talbot CE, Jeffery ND. Findings on low-field cranial MR images in epileptic dogs that lack interictal neurological deficits. Vet J. 2008; 176:320-325. [PubMed: 17499532]

65. Hu H, Barker A, Harcourt-Brown T, Jeffery N. Systematic review of brain tumor treatment in dogs. J Vet Intern Med. 2015; 29:1456-1463. [PubMed: 26375164]

66. Dickinson PJ. Advances in diagnostic and treatment modalities for intracranial tumors. J Vet Intern Med. 2014; 28:1165-1185. [PubMed: 24814688]

67. Eisele SC, Wen PY, Lee EQ. Assessment of brain tumor response: RANO and its offspring. Curr Treat Options Oncol. 2016; 17:35. [PubMed: 27262709]

68. Brandao LA, Shiroishi MS, Law M. Brain tumors: A multimodality approach with diffusionweighted imaging, diffusion tensor imaging, magnetic resonance spectroscopy, dynamic susceptibility contrast and dynamic contrast-enhanced magnetic resonance imaging. Magn Reson Imaging Clin N Am. 2013; 21:199-239. [PubMed: 23642551]

69. Van Meervenne S, Verhoeven PS, de Vos J, Gielen IM, Polis I, Van Ham LM. Comparison between symptomatic treatment and lomustine supplementation in 71 dogs with intracranial, spaceoccupying lesions. Vet Comp Oncol. 2014; 12:67-77. [PubMed: 22738696]

70. Heidner GL, Kornegay JN, Page RL, Dodge RK, Thrall DE. Analysis of survival in a retrospective study of 86 dogs with brain tumors. J Vet Intern Med. 1991; 5:219-226. [PubMed: 1941756]

71. Bley CR, Sumova A, Roos M, Kaser-Hotz B. Irradiation of brain tumors in dogs with neurologic disease. J Vet Intern Med. 2005; 19:849-854. [PubMed: 16355679] 
72. Mariani CL, Schubert TA, House RA, et al. Frameless stereotactic radiosurgery for the treatment of primary intracranial tumours in dogs. Vet Comp Oncol. 2015; 13:409-423. [PubMed: 24007303]

73. Hansen KS, Zwingenberger AL, Theon AP, Pfeiffer I, Kent MS. Treatment of MRI-diagnosed trigeminal peripheral nerve sheath tumors by stereotactic radiotherapy in dogs. J Vet Intern Med. 2016; 30:1112-1120. [PubMed: 27279132]

74. Dickinson PJ, LeCouteur RA, Higgins RJ, et al. Canine spontaneous glioma: a translational model system for convection-enhanced delivery. Neuro Oncol. 2010; 12:928-940. [PubMed: 20488958]

75. Rossmeisl JH Jr, Garcia PA, Pancotto TE, et al. Safety and feasibility of the NanoKnife system for irreversible electroporation ablative treatment of canine spontaneous intracranial gliomas. $\mathrm{J}$ Neurosurg. 2015; 123:1008-1025. [PubMed: 26140483]

76. Albert FK, Forsting M, Sartor K, Adams HP, Kunze S. Early postoperative magnetic resonance imaging after resection of malignant glioma: objective evaluation of residual tumor and its influence on regrowth and prognosis. Neurosurgery. 1994; 34:45-60. discussion 60-1. [PubMed: 8121569]

77. Simpson D. The recurrence of intracranial meningiomas after surgical treatment. J Neurol Neurosurg Psychiatry. 1957; 20:22-39. [PubMed: 13406590]

78. Brown TJ, Brennan MC, Li M, et al. Association of the extent of resection with survival in glioblastoma: A systematic review and meta-analysis. JAMA Oncol. 2016; 2:1460-1469. [PubMed: 27310651]

79. Cameron S, Rishniw M, Miller AD, Sturges B, Dewey CW. Characteristics and survival of 121 cats undergoing excision of intracranial meningiomas (1994-2011). Vet Surg. 2015; 44:772-776. [PubMed: 26032173]

80. Meij BP. Hypophysectomy as a treatment for canine and feline Cushing's disease. Vet Clin North Am Small Anim Pract. 2001; 31:1015-1041. [PubMed: 11570124]

81. van Rijn SJ, Galac S, Tryfonidou MA, et al. The influence of pituitary size on outcome after transsphenoidal hypophysectomy in a large cohort of dogs with pituitary-dependent hypercortisolism. J Vet Intern Med. 2016; 30:989-995. [PubMed: 27425149]

82. Brundage CM, Packer RA, Jones MD. Magnetic resonance imaging appearance and mechanism of action of five hemostatic agents used in neurosurgery. Vet Surg. 2016; 45:996-1004. [PubMed: 27658949]

83. Sinclair AG, Scoffings DJ. Imaging of the post-operative cranium. Radiographics. 2010; 30:461482. [PubMed: 20228329]

84. Chow KE, Tyrrell D, Long SN. Early postoperative magnetic resonance imaging findings in five dogs with confirmed and suspected brain tumors. Vet Radiol Ultrasound. 2015; 56:531-539. [PubMed: 26372362]

85. Forsyth PA, Petrov E, Mahallati H, et al. Prospective study of postoperative magnetic resonance imaging in patients with malignant gliomas. J Clin Oncol. 1997; 15:2076-2081. [PubMed: 9164220]

86. Akeson P, Vikhoff B, Stahlberg F, Holtas S. Brain lesion contrast in MR imaging. Dependence on field strength and concentration of gadodiamide injection in patients and phantoms. Acta Radiol. 1997; 38:14-18. [PubMed: 9059395]

87. Nobauer-Huhmann IM, Ba-Ssalamah A, Mlynarik V, et al. Magnetic resonance imaging contrast enhancement of brain tumors at 3 Tesla versus 1.5 Tesla. Invest Radiol. 2002; 37:114-119. [PubMed: 11882790]

88. Chang KH, Ra DG, Han MH, Cha SH, Kim HD, Han MC. Contrast enhancement of brain tumors at different MR field strengths: Comparison of $0.5 \mathrm{~T}$ and 2.0 T. AJNR Am J Neuroradiol. 1994; 15:1413-1419. discussion 1420-3. [PubMed: 7985558]

89. Lescher S, Schniewindt S, Jurcoane A, Senft C, Hattingen E. Time window for postoperative reactive enhancement after resection of brain tumors: Less than 72 hours. Neurosurg Focus. 2014; 37:E3.

90. Therasse P, Arbuck SG, Eisenhauer EA, et al. New guidelines to evaluate response to treatment in solid tumors: European Organization fro Research and Treatment of Cancer, National Cancer Institute of the United States, National Cancer Institute of Canada. J Natl Cancer Inst. 2000; 92:205-216. [PubMed: 10655437] 
91. Eisenhauer AE, Therasse P, Bogaerts J, et al. New response evaluation criteria in solid tumours: Revised RECIST guideline (version 1.1). Eur J Cancer. 2009; 45:228-247. [PubMed: 19097774]

92. Quant EC, Wen PY. Response assessment in neuro-oncology. Curr Oncol Rep. 2011; 13:50-56. [PubMed: 21086192]

93. Butowski N, Chang SM. Endpoints for clinical trials and revised assessment in neuro-oncology. Curr Opin Neurol. 2012; 25:780-785. [PubMed: 23007010] 


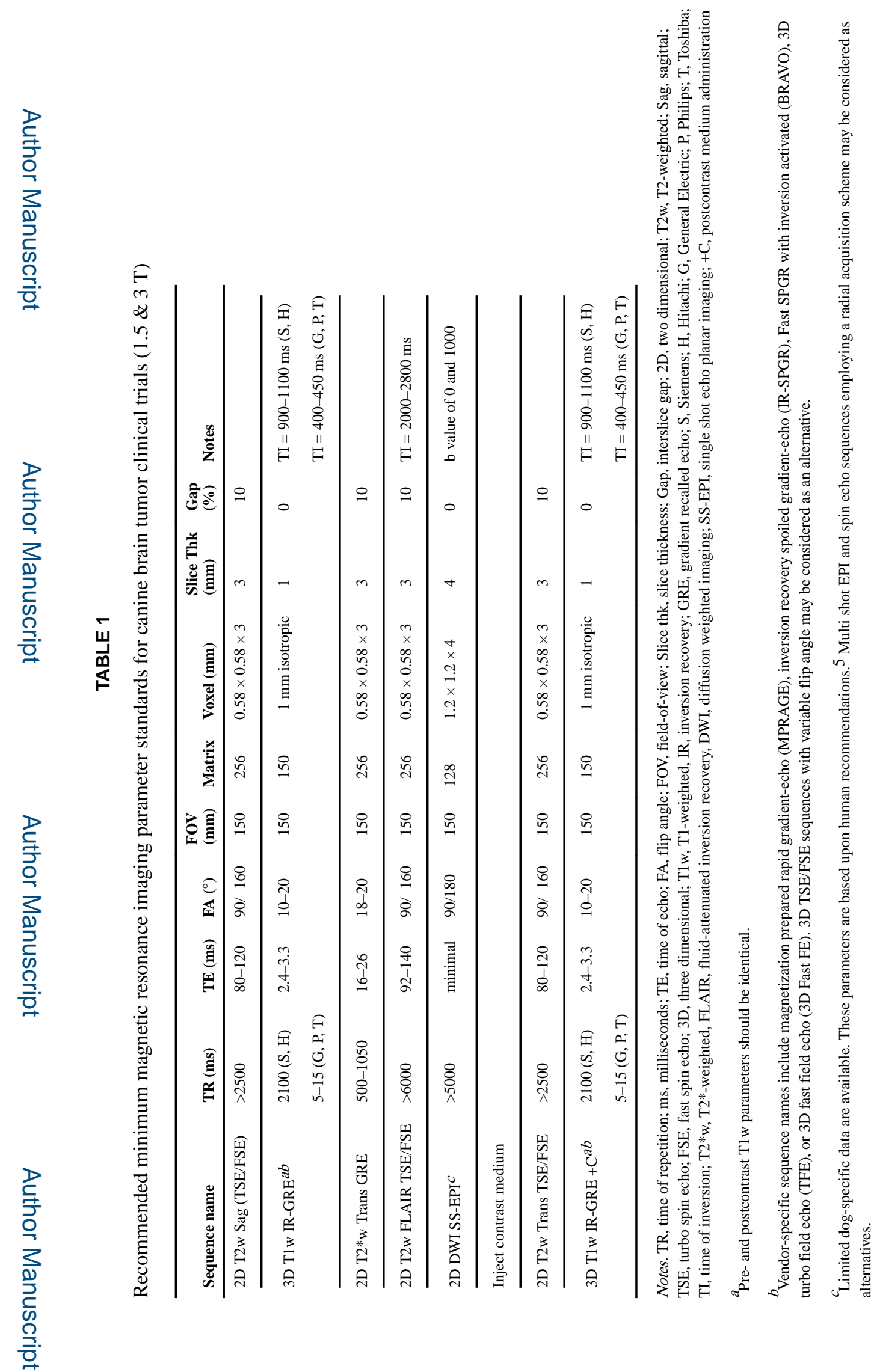

Vet Radiol Ultrasound. Author manuscript; available in PMC 2019 May 01. 
TABLE 2

Recommended early post-operative timing for magnetic resonance imaging

\begin{tabular}{llll}
\hline Tesla & $\begin{array}{l}\text { Initial } \\
\text { postoperative } \\
\text { imaging }\end{array}$ & $\begin{array}{l}\text { Subseqent } \\
\text { imaging }\end{array}$ & Thereafter \\
\hline$<3$ T MR unit & $<72$ hours postoperatively & 6 weeks posttreatment & Q 3 months serially \\
\hline 3T MR unit & $<24$ hours postoperatively & 6 weeks posttreatment & Q 3 months serially \\
\hline
\end{tabular}

Note. MR, magnetic resonance. 


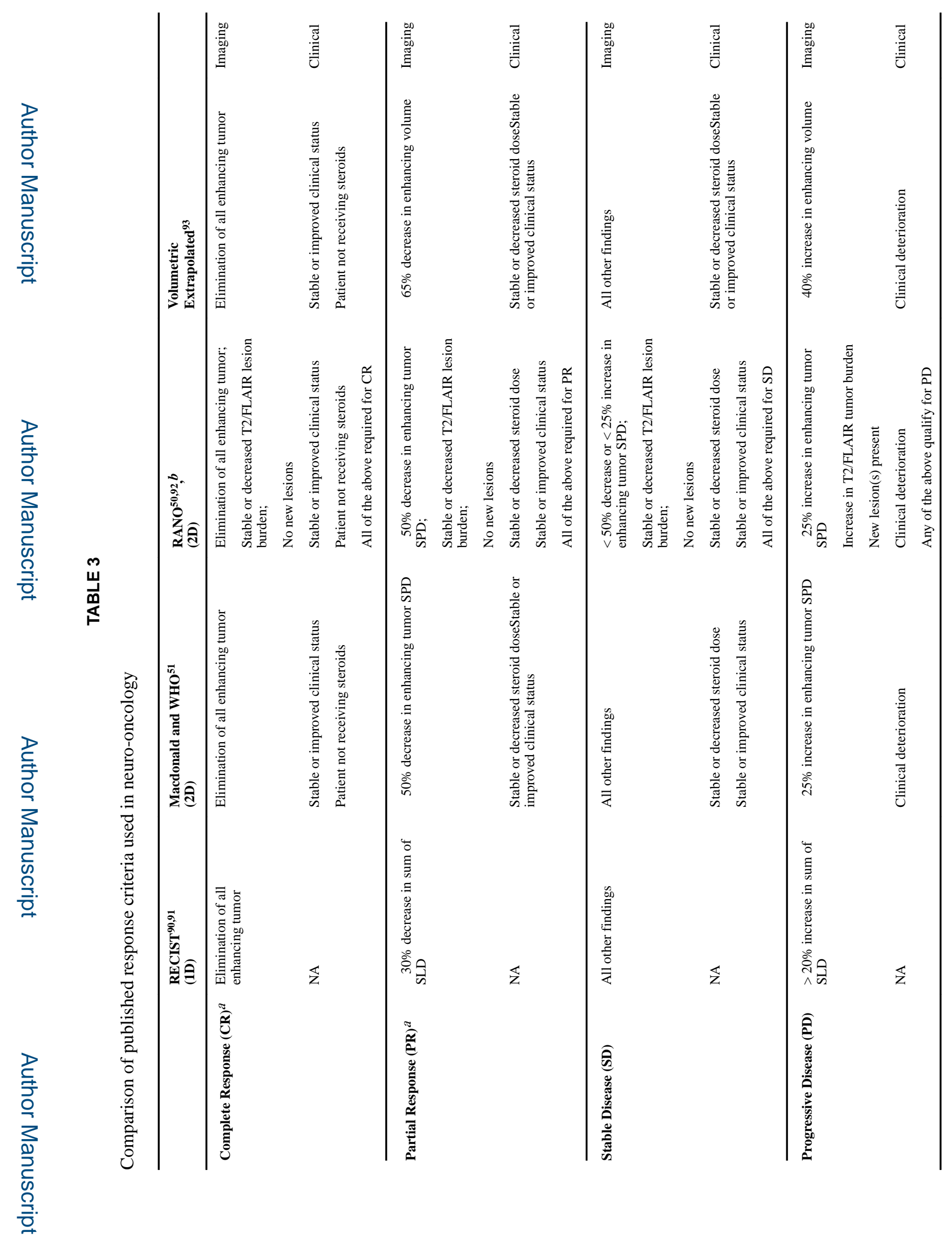

Vet Radiol Ultrasound. Author manuscript; available in PMC 2019 May 01. 


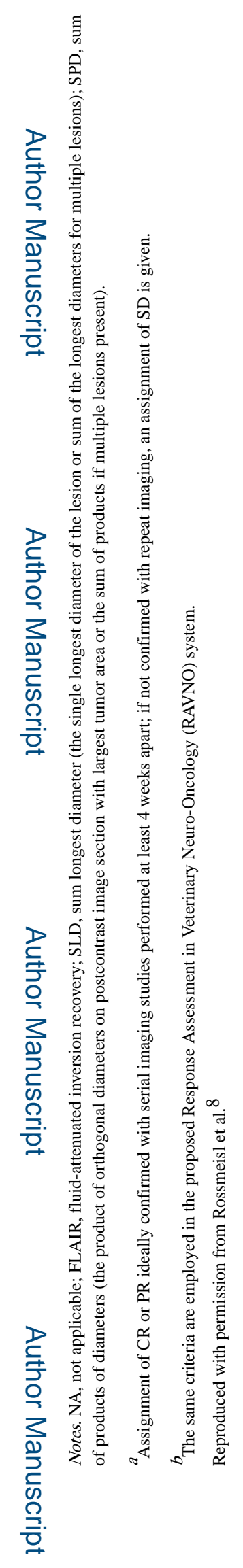

Vet Radiol Ultrasound. Author manuscript; available in PMC 2019 May 01. 